\title{
E Health and M health: Future of Healthcare
}

\author{
Abas Khan ${ }^{1}$ and Mohammed Sarwar Mir*2 \\ ${ }^{1}$ Senior Resident, Department of Hospital administration, India \\ ${ }^{2}$ Resident Medical Officer, SKIMS, India \\ *Corresponding author: Mohammed Sarwar Mir, Resident Medical Officer, SKIMS, India
}

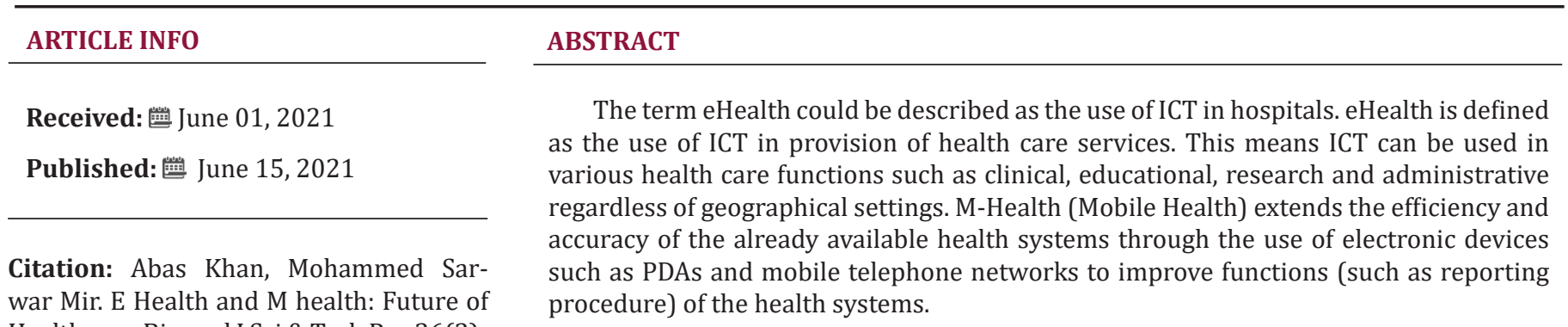

Keywords: e Health; m Health; Health Care

\section{Background}

The term eHealth could be described as the use of ICT in hospitals. eHealth is defined as the use of ICT in provision of health care services. This means ICT can be used in various health care functions such as clinical, educational, research and administrative regardless of geographical settings. M-Health (Mobile Health) extends the efficiency and accuracy of the already available health systems through the use of electronic devices such as PDAs and mobile telephone networks to improve functions (such as reporting procedure) of the health systems. ICT can be used to transform the health paradigm by shifting the provider-patient configuration this typically can involve extending health care services to underserved areas by use of electronic or telecommunication means (Telemedicine) such as video chat, or health telephone hotlines. This arrangement provides patients with seamless access to doctors, improved diagnosis and treatment around the clock. E-Health and m-Health can transform the health systems by incorporating electronic means to deliver information and provide health related training. For example, mobile phones can be used to disseminate information regarding vaccination campaign and the Internet can be used to provide distance learning, share information etc. M-Health occur mostly through mobile provision of health care services. It occurs through wireless telemedicine that involve the use of mobile telecommunications and multimedia technologies and their integration with mobile health care delivery systems. M-Health continues to mature and has been used to address health care challenges such as access, quality, affordability, matching of resources and behavioural norms through mobile technologies. M-Health is a network involving people and products; and the mechanisms that connect them using digital technologies. E-health technologies and processes such as individual electronic health records, clinical decision support systems and intelligent, responsive buildings and equipment have enabled the delivery of safe, high quality healthcare. The culture of healthcare has evolved substantially from the days where mistakes, errors, omissions and duplication were common in healthcare delivery. This restructuring entails a two-fold paradigm shift:

a) From symptom-based to preventive healthcare and

b) From hospital-centred to person-centred health systems.

\section{E-Health Challenges Especially in Developing Countries}

Not everyone is singing the praises of ICT in health education and practice. Coyne (1995) argued that the implementation of ICT can lead to a widening of the gap between rich and poor, as well as of the "digital divide", described by World Health Organization (WHO) as being "more dramatic than any other inequity in health or income". 
Lack of Skilled Stakeholders: One of the challenges to eHealth implementation in developing countries is the lack of skilled users. The users that lack skills in using the system are health practitioners or patients, developers and maintainers, related ICT professionals, these maybe due to poor literacy and poor technological skills internet and computer literacy.

The

i. Stakeholders with low level of education are technical staff but are the primary users of the Health Information Systems

ii. In developing countries. A major challenge is training users in patient confidentiality.

Technical and Operational: Implementation of electronic health needs strong relations between ICT and Information Systems in various organizations, which has its own complexity. Some of the problems and facing challenges in implementing E-health, in Developing Countries are, from the technical and operational points of view, regarded as follows:

i. Lack of a proper framework for information quality characteristics
ii. Needing suitable medical equipment
iii. Movement and Transportability
iv. Losing and missing electronic health records
v. Maintenance, supporting and updating the project

Social and Cultural: Developing electronic health in the society has its own peculiarities which are quite important due to the cultural and social limitations in Developing Countries. Some of the problems in this field are indicated as follows:

i. Lack of widespread and continuous education for public use of E-health services

ii. The need for nationalizing the health culture in involved areas in developing Telemedicine

iii. Cultural limitations against executing E-health services

iv. Resistance against changes due to habits

v. Inadequate knowledge levels of people with regards to using E-health

Legal: Definitely, one of the most important factors related to allocation and development of E-health is provision of legal aspects and balancing whole collection of laws and regulations, with this phenomenon. Some of the legal challenges of electronic health system, in most countries especially Developing Countries, are as follows:

i. Lack of following government's ratified laws ii. No support of national and universal standards

iii. Lack of existing suitable laws regarding personal rights and keeping patients' private surroundings

iv. The need for developing a legal and lawful framework for managing it in health care

v. The need for developing a framework for transmitting inhomogeneous data and unifying them

Financials: Management limitations in using workforce, physical and financial sources and the necessity for the managers to have systematic approach and concentrating processes productivities during operation of most resources that they have authority upon, increase the importance of existing Information Systems and their operating mechanisms. Hence, considering the following challenges is a part of management responsibilities in this field:

i. The need for investment and allocation of regular budget in electronic health field and using relevant technologies in health section

ii. Unsettled increase of healthcare costs

iii. Lack of framework for economic analysis of benefits and the results of remote health control

iv. Lack of consideration of financial and operative situations of each of the host countries, separately

Cultural Hindrances: At its core, the healthcare industry is built on care providers using legacy technology and rigid processes, tightly holding onto data to remain within the Privacy Act. Subsequently, the largest hurdle to be faced is changing perceptions on data sharing as a whole, to foster a true culture of integration. This means that the data generated, while managed and protected, still needs to be shared among authorized entities [1-10].

\section{Types of eHealth Solutions}

Self-Management: ICT tools used by patients to manage their health more independently. Self-management tools include computerized systems - e.g. computers, tablets, mHealth, wearable devices or other assistive technologies - which promote care management and provide health advice and reminders. Also, specific tools for informal carers to co-manage care activities or to get direct support for their own psychological, emotional or social needs.

Remote Consultation, Monitoring and Care: ICT tools used for providing and enhancing the remote interaction between patients and health professionals. Remote eHealth solutions include the consultations and visits at a distance that are typical of telehealth and telemedicine services, as well as continuous monitoring of specific conditions, behaviours and safety (telemonitoring and 
telecare systems). Specific tools can also improve communication, such as online scheduling of clinical appointments, ePrescriptions and direct communication with health care staff.

Health Care Management: ICT tools are used for improving the integration, quality and efficiency of care processes within and between care providers. Such eHealth tools include EHRs and health information systems for their registration, reproduction and sharing between professionals, eventually allowing the individual to access and use them as PHRs. Furthermore, ICT tools can improve the speed and consistency of collaboration and communication between care professionals involved in health care.

Health Data Analytics: ICT tools based on information systems that exploit and analyze data in patient databases and/ or clinical evidence for prevention, monitoring or treatment purposes. DSSs are used by health professionals to improve clinical decisionmaking in individual complex cases. Risk stratification systems monitor the health data of a regional or national population, with the possibility of identifying patients with specific health risks, which may have the potential to support preventative, monitoring or treatment strategies as necessary.

\section{ISSN: 2574-1241}

DOI: 10.26717/BJSTR.2021.36.005864

Mohammed Sarwar Mir. Biomed J Sci \& Tech Res

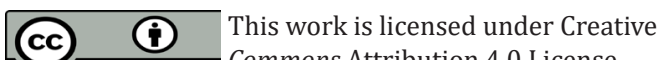

Submission Link: https://biomedres.us/submit-manuscript.php

\section{References}

1. D Roberts (2012) No Brainer - Commercial Buildings Should Save Money by Becoming More Energy Efficient.

2. (2017) Energy star. What is energy use intensity (EUI).

3. S Alhurayess, MK Darwish (2012) Analysis of energy management in hospitals. In $201247^{\text {th }}$ International Universities Power Engineering Conference (UPEC), p. 1-4.

4. B Schiller (2012) Most energy-efficient hospitals in the country.

5. M Brambley, D Hansen, P Haves, D Holmberg, S McDonald, et al. (2005) Advanced sensors and controls for building applications: market assessment and potential R\&D pathways, PNNL-15149, Technical report, Prepared for the U.S. Department of Energy by Pacific Northwest National Laboratory 2005.

6. (2014) ISO 16484-5. Building automation and control systems (BACS) Part 5: Data communication protocols.

7. (2006) ISO/IEC 14543-3-1. Information Technology - Home Electronic Systems (HES) Architecture - Part 3-1: Communication layers Application layer for network based control of HES Class 1.

8. (2016) Mercy Virtual Care Center.

9. (2016) Bilişim Zirvesi, Akıllı Hastane.

10. Wei dong WANG (2004) The digital hospital in future: understanding and management of our future hospital. Information of Medical Equipment, p. 7.

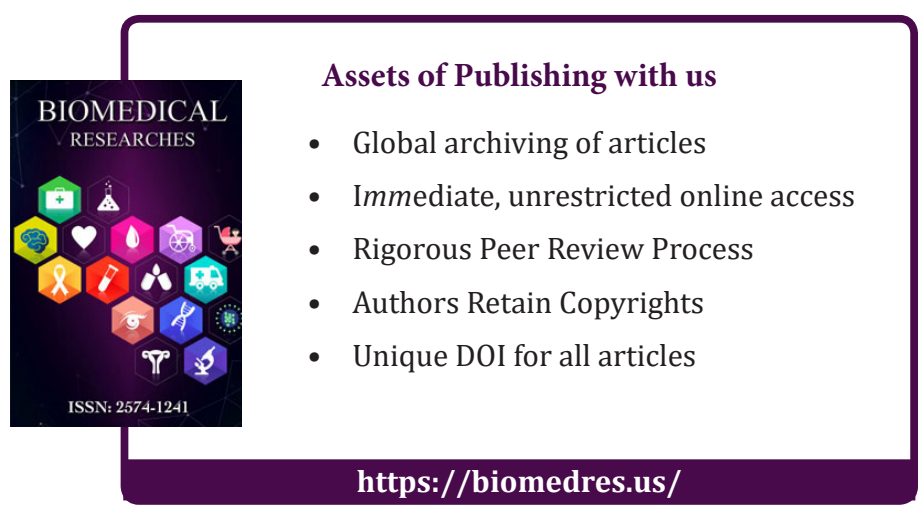

